\title{
Research and Performance of Recognition System of the Human Activity with a Filter Bank of Gabor by Hidden Markov Model
}

\author{
Rajeev Shrivastava
}

\begin{abstract}
-recognition of human movement is one of the huge growing generation. It has a massive feature for example supervision (movements evaluation), safety (walker detection), manage (character-computer interfaces); content material- based video retrieval, and plenty of others. Human interest reputation device of is a device of identifying a selection of Human sports activities beside a few saved sample Human interest.

In this paper Human activity reputation machine for popularity of man or woman is provided. It gets facts of individual photo and look for comparable interior the store pics. Human interest can be visible as fit or now not fit if there can be in shape or not matched in stop result. consumer cannot create a few form of regulate inside the stored photo documents, i.e. a purchaser isn't always accredited to insert or dispose of photographs from the garage records. The manager of the scheme has verification to make changes in the storage facts. The supervisor of the scheme has verification to make adjustments inside the storage statistics. Biometrics device of automatic Human hobby recognition system acting recognition is supplied. Extraction of capabilities is finished through the usage of the use of Gabor filter out to this tool. function extraction of the picture is convolving with Gabor clear out and extra person pattern era set of guidelines is used to determine a hard and rapid of realistic and non redundant functions of Gabor. Hidden Markov models for matching the input Human interest photograph to the stored pics is used.
\end{abstract}

Keywords: HMM, Gabor, KTH

\section{INTRODUCTION}

It is difficult to recognize automatic human activity in computer vision .First goal is to understand human visual system which is complex. It includes knowledge to represent human activity. This is essential in order to differentiate betweendiverse identities by highcorrectness. Two essential with theoretically autonomous difficulties have to be tackled in this type of systems namely human activity exposure as well as the identification of the detected human activity. Effort on the identification phase, obtain the detected human activity standards as input to the algorithm. This phase can be divided into two steps: extraction of a feature, where significant information for inequity is accumulated and the identical pace, where the identification answer is given with the help of a human activity database.

In the route of this prevent, numerous strategies may be created inside the literature [9]. a few strategies are based totally on holistic frame records in which no attempt is made to perceive person frame factors. Authors like [10] utilize Hidden Markov model and AdaBoost for identification of three-D character motion in view of joint pose or

Revised Manuscript Received on April 12, 2019.

Dr Rajeev Shrivastava,(HOD St. Martin's Engg. College)Secunderabad, Telangana, India.(rajeev2440130@gmail.com) characteristic method. no matter the truth that, there are moves which can be superior acquainted thru the usage of merely considering body divisions, which includes the dynamics of the legs for on foot, walking and taking walks [4]. consequently, motion identification may be based totally on a previous recognition of the human frame components [9]. In this case, human body divisions ought to be first of all detected within the photograph: writers similar to $[8,11]$ explains human recognition algorithms via probabilistic body department accumulating. author [12-19] labored in reputation and enhance accuracy fee.

I've already used this concept in face reputation [15] and this paper extends version of this idea this newsletter is ready as follows. In segment II we've were given were given provided hassle definition and proposed a solution in element. section III explains the picture Processing. section IV describes the image Modeling. phase $\mathrm{V}$ explains the tentative information and outcomes. in the end, conclusions and destiny direction are drawn in section VI.

\section{DEFINITION OF PROBLEM AND PROJECTED RESULT}

\subsection{Definitionof Problem}

Recognition of Automatic human activity is a troublesome part in computer vision. One of the key objectives is to understand complex human visual system .Along with this it is essential to know represention of human activity. This is done in order to distinguish between different identities with elevated correctness. Two problems which are fundamentally and theoretically independents are to be addressed bu this type of scheme:

1. Human activity detection

2. To recognize the detected human activity.

Art work on the identification segment, taking the detected human interest due to the fact the enter to the set of rules. This phase can be divided in steps: 1. feature extraction, wherein big information for inequity is stored, and 2. matching step, in which the recognition very last outcomes is given with the assist of a human hobby report

\section{2 endorsed answer}

We used Gabor easy out for choosing Gabor capabilities for recognition of human interest. A tiny department of functions of Gabor in a function of discriminating from

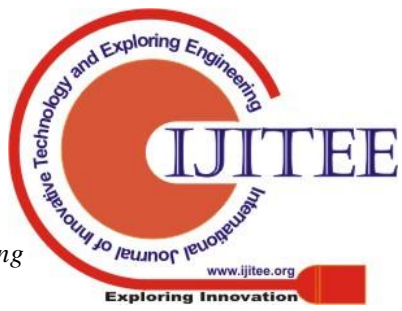




\section{Research And Performance Of Recognition System Of The Human Activity With A Filter Bank Of Gabor By Hidden Markov Model}

proper human interest images that is stored within the document. in this paper the method advanced uses the hidden Markov model to healthy a check human interest photograph with a appropriate reference photo.

From the given human interest picture Gabor easy out is utilized by this tool to extract the gathering of useful Gabor functions. The acquired abilities are over again finished to discrete Radon redecorate to cast off a sequence of feature vectors from an picture. The HMM-based totally scheme superior indoors this article suits the feature set (exam series) designed for an exam picture with a Hidden Markov version of the accumulated image, through Viterbi alignment [3].

via way of calculating terrible $\log$ threat a distance evaluates is won. [2]\& [5].

\subsubsection{Design of System}

1. Extraction of feature. The systems expanded in this article use similar feature extraction techniques. The bulk of the image dealing out and extraction of feature engages the calculation of the Discrete Radon Transform of each image. [6] The DRT is attained by scheming projections of every image at diverse directions.Subsequent to a few additional processing of images (normalization), each of these projections constitutes a feature vector in an examination series. These features are classified as global features since they are not extracted at stroke or sub-stroke level.

2. Image modeling. The systems developed in this dissertation use two very different approaches to model a specific human activity image .In the case of system, based on the HMM, each facial image is modeled through an HMM of which the positions be ordered in a circle in this paper HMM based system is advanced [8].

3. Matching. The distance between tests images with a model used for the claimed image is attained as follows. Through Viterbi alignment, the system based on HMM expanded in this article test the set of feature (study series) for a test image with an HMM of the particular image. A calculate of distance is attained through calculating negative $\log$ possibility.

4. Verification.on the equal time as a declare is made that a check photograph belongs to a particular man or woman the extracted statement collection is first matched with a version of the photo in order that a distance calculated is obtained. This distance measure is then normalized so that you can seize up on the version in the human interest image. The version inside the human interest photo is expected with the aid of matching all the education pics with the photo model. in this manner, numerous distance measures are received. statistics of these distance measures are then used to estimate the version in the image education set. A international threshold, that may be a threshold it is the identical for all pics, can, therefore, be used. check images, for which the normalized distance diploma is an lousy lot much less than this threshold, are general? The others are rejected. A plan instance of the systems superior in this paper is given in determine 2.1.

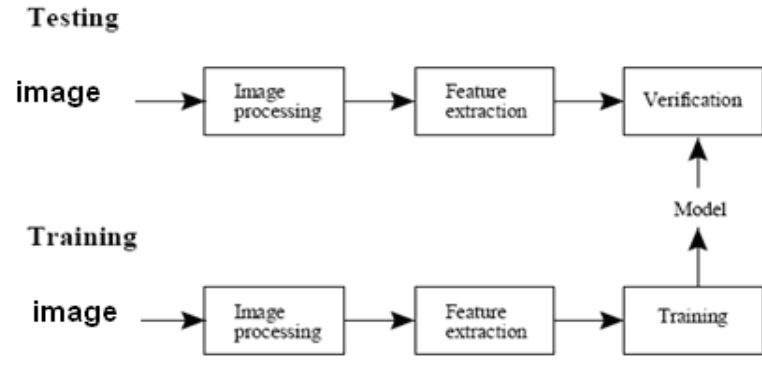

Fig 2.1: A schematic representation of the systems.

\subsection{Filter Bank Construction}

We will have to consider a range of Gabor filters with equal scales and orientations . This is essential in order to effectively envelop the frequency spectrum of Filter Bank Construction. The motive is to give sufficient exposure to the frequency components of importance as continue a least of overlie among filters so as to get a compute of autonomy among the taken outcoefficient.

\subsection{Gabor filter}

In the spacial area Gabor filter, the two dimensions Gabor filter modulated by a sinusoidal plane wave is a Gaussian kernel

$\phi \Pi(\mathrm{f}, \theta, \gamma, \eta)(\mathrm{x}, \mathrm{y})$

$$
=\left(f^{2} / \pi \gamma \eta\right) e^{-\left(\alpha^{2} x^{\prime 2}+\beta^{2} y^{\prime 2}\right)} e^{j 2 \pi f x^{\prime}}
$$

$\mathrm{x}^{\prime}=\mathrm{x} \cos \theta+\mathrm{y} \sin \theta$

$\mathrm{y}^{\prime}=-\mathrm{x} \sin \theta+\mathrm{y} \cos \theta$,

where $f$ (cycles/pixel) is the primary frequency of the sinusoidal aircraft wave, $\theta$ Are the anticlockwise rotation of the Gaussian and the plane wave, $\alpha$ Is the sharpness of the Gaussian along the primary axis just like the wave, and $\beta$ Is the sharpness of the Gaussian minor axis perpendicular to the wave. $\gamma=\mathrm{f} / \alpha$ and $\eta=\mathrm{f} / \beta$ are extremely good such that the ratio amongst frequency and sharpness is ordinary

2.5. example of Gabor feature

Filters have been deliberate; image functions at various positions, density, and commands may be taken out through convolving the image $\mathrm{I}(\mathrm{x}, \mathrm{y})$ with the filters:

OП $(\mathrm{f}, \theta, \gamma, \eta)(\mathrm{x}, \mathrm{y})=\mathrm{I}(\mathrm{x}, \mathrm{y}) * \phi \Pi(\mathrm{f}, \theta, \gamma, \eta)(\mathrm{x}, \mathrm{y})$. some of Gabor filters at numerous levels and instructions are frequently used. A smooth out economic institution via eight $* 8$ and 8 orientations for feature extraction is designed.

\section{PROCESSING OF IMAGE}

Every image is examined into a dual image on a resolution of three hundred points for every inch when that median filtering is useful to get rid of speckle noise. The image dimensions don't seem to be normalized. Later, the DRT of every image is considered. Every line of the DRT symbolizes an extension or shade of the image at an explicit direction. When these projections are practiced and normalized, they symbolize a collection of featurevectors (examinationseries) used for the image in a query. The DRT of a picture is estimated as pursues. 
Assume that every photo consists of Pixels in whole, which the intensity of the ith detail is signified through Ii, $\mathrm{i}=1, \ldots, \Psi$. The DRT is taken into consideration exploitation $\beta$ non overlapping beams constant with attitude and $\Theta$ angles in general. The additive intensity of the pixels that lie most of the jth beam is symbolized thru $\mathrm{Rj}, \mathrm{j}=1, \ldots$ ., $\beta \Theta$. this could be referred to as the jth beam upload. In its top notch type, the atomic variety 86 remodels is probably expressed.

The correctness of the DRT is ready thru using $\Theta$ (the style of angles), $\beta$ (the form of beams consistent with angle), along with the correctness of the interpolation approach. reminder to the persistent form of the atomic quantity 86 remodels is inverted within the path of analytical indicates that the DRT so includes in reality a similar statistics because of the reality the specific photograph is probably with overall performance calculated with companion in algorithmic rule through Brace nicely [1]. Our scheme measures the DRT at $\Theta$ angles. the ones angles are evenly disbursed amongst $0 \circ$ and one hundred eighty ${ }^{\circ}$.

The dimension of every projection is later modified from $\beta$ to $d$. this can be completed through manner of preliminary decimating all the 0 -valued additives starting every projection. the ones decimated vectors are in some time contracted or distended to a period of $\mathrm{d}$ sooner or later of interpolation. despite the fact that maximum the records within the unique photograph is limited in the projections at instructions that alternate from zero ${ }^{\circ}$ to a hundred $80^{\circ}$, the projections at recommendations that range from 100 and eighty $^{\circ}$ to $360^{\circ}$ additionally be encircled within the examination collection. those in addition projections are greater to the exam collection at the way to create certain that the gathering suits the topology of our HMM.

On the equal time as these projections are certainly reflections of the projections previously deliberate, no further calculations are critical.

Associate in Nursing examination series so consists of $\mathrm{T}=2 \Theta$ feature vectors, that is, $\mathrm{XT} 1=\{\mathrm{x} 1, \mathrm{x} 2 \ldots \mathrm{xT}\}$. Every vector is later normalized by the difference of the intensity of the whole set of $\mathrm{T}$ feature vectors. Every image pattern is so diagrammatic by Associate in observation sequence that consists of $\mathrm{T}$ examinations, wherever every observation could be a feature vector of measurement $d$. The Discrete Randon Transform, as an extraction feature method, has many blessings. Though the DRT isn't a shift invariant illustration of an image, shift and scale exchangeability is ensured by the next image process. Every image could be astill image and holds no active data. As the feature vectors are gained by scheming projections at totally dissimilar angles, simulated time evolution is formed from one feature vector to following, every time the angle is that the dynamic changeable This allows USA to make Associate in Nursing HMM for every image. The DRT be computed on Associate in which phase varies from $0^{\circ}$ to $360^{\circ}$ and every examination series is next shapely through an HMM of that the positions are systematized during a ring. This guarantees that rotation invariant is in every set of feature vectors.

This scheme is additionally sturdy with regard to moderate levels of noise

\section{MODELINGOF IMAGES USING HMM}

HMM-based scheme utilizes endless initial arrangements, HMM to signify every image. The HMM-based and DTWbased schemes use same authentication procedures. A pattern recognition scheme that relies on HMMs usually employs Associate in Nursing HMM to signify every model category. Every of those HMMs is employed to model Associate in Nursing examination series, similarly because the relationship involving the individual examinations. HMMs are so created in such how that time-evolution is supposed from one examination within the series to subsequent. While speech signals and dynamic (on-line) pictures conjointly have temporal data, it's potential to remove endless examination series from these signals in a extremely intuitive method. For this cause HMMs are particularly well-matched for modeling these forms of signals. This can be not the case for static (off-line) pictures. as a result, feature vectors got to be take oute from off-line pictures in such how that time-evolution is simulated from one examination to subsequent.

In this newsletter hire a grid to segment a picture into local sq. cells. From each mobile, the element mass is calculated, so that it will each detail density represent a neighborhood feature. each picture is so diagrammatic by using a chain of characteristic vectors, anyplace each feature vector signifies the detail densities associated with a column of cells. The HMM-supported corporation advanced simulates time-evolution from one examination to next by means of scheme the DRT of each image all through the function extraction approach preceding to we tend to communicate the HMM-based totally photograph representation, we have a tendency to preliminary present the notation within the subsequent component.

The HMM-supported organization developed within simulates time-evolution from one examination in partner in exam collection to next with the aid of scheme the Discrete Randon rework of a rare image. The characteristic vectors are so attained by way of scheming projections of an image at absolutely various directions, when that they may be subjected to some greater process. The course is so the dynamic variable. This permits united states of america to create companion in Nursing HMM for every photo. 
Research And Performance Of Recognition System Of The Human Activity With A Filter Bank Of Gabor By Hidden Markov Model

\section{EXPERIMENTAL DATA AND RESULTS}

Results \& Discussion

\subsection{Screen Shots}

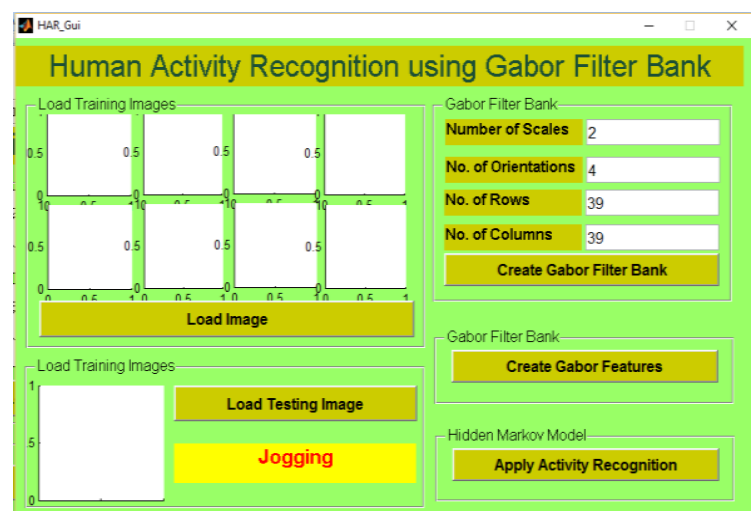

Figure 5.1: GUI Showing the Control Panel for implementation of the proposed work

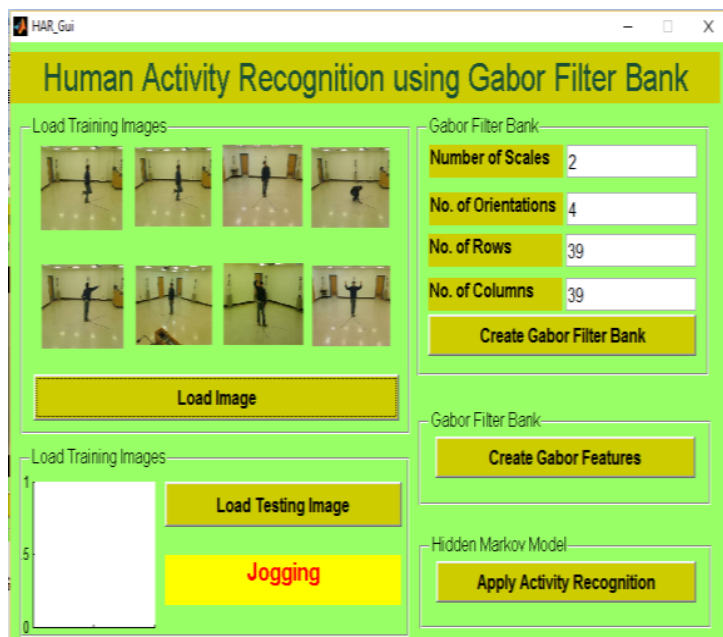

Figure 5.2: GUI Showing the Control Panel Loaded with Image and Parameters of the Filter Bank of Gabor

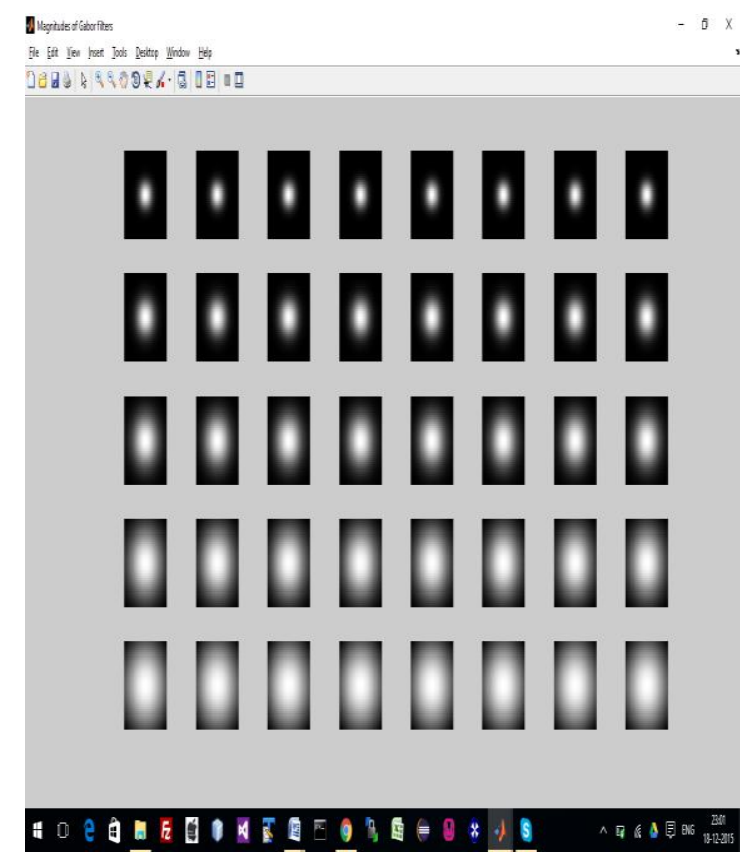

Figure 5.3:GUI Showing the Filter Bank of Gabor with 5 Scales and 8 Orientations

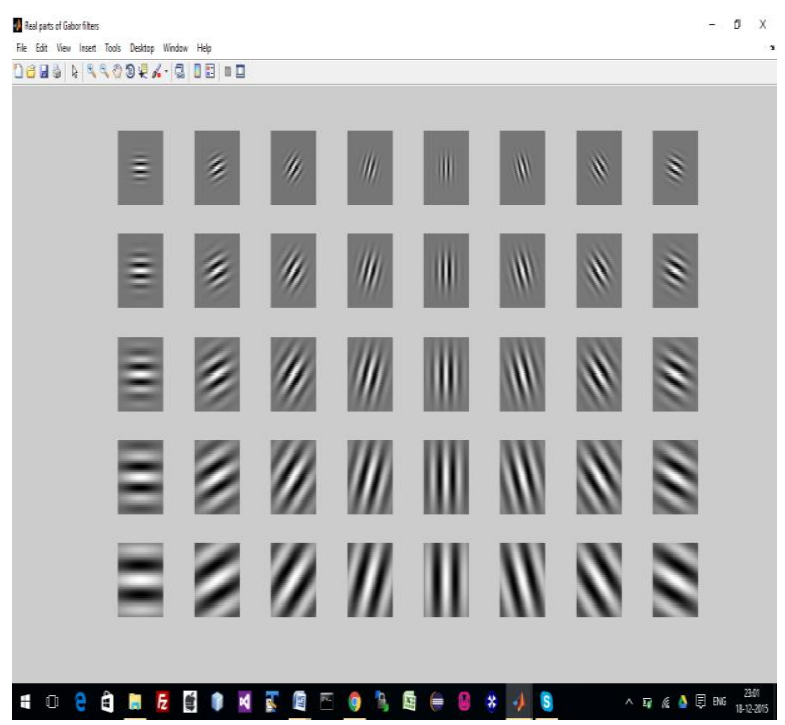

Figure 5.4:GUI Showing the Filter Bank of Gabor with 5 Scales and 8 Orientations

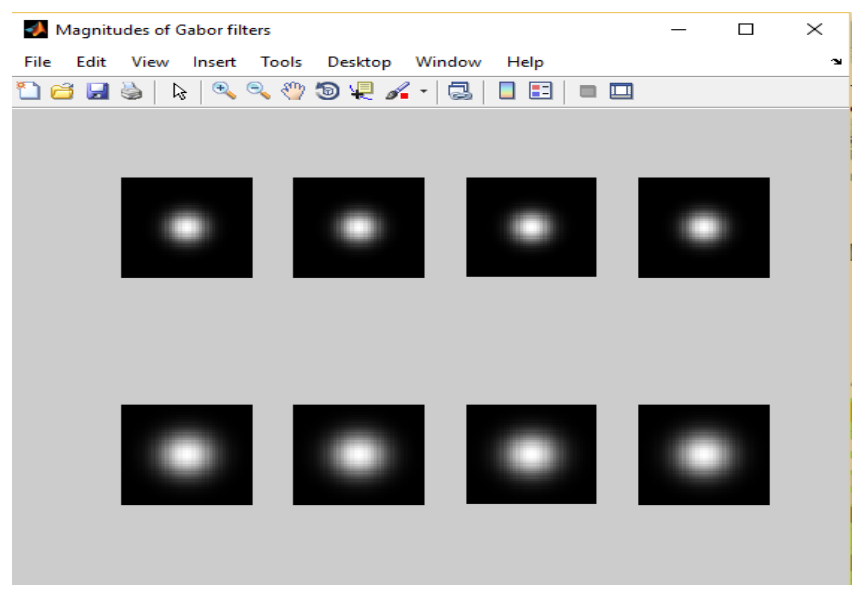

Figure 5.5: GUI Showing the Filter Bank of Gabor with 2 Scales and 4 Orientations

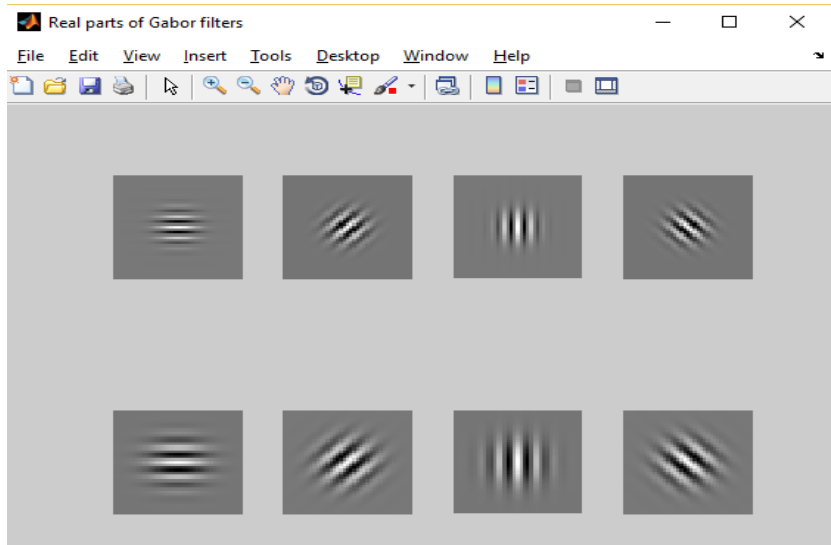

Figure 5.6: GUI Showing the Filter Bank of Gabor with 2 Scales and 4 Orientations

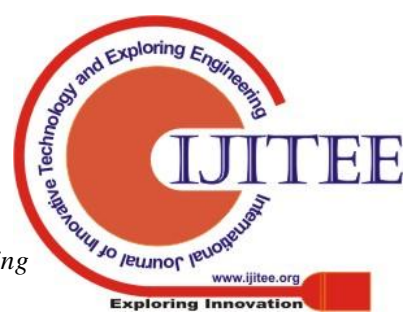




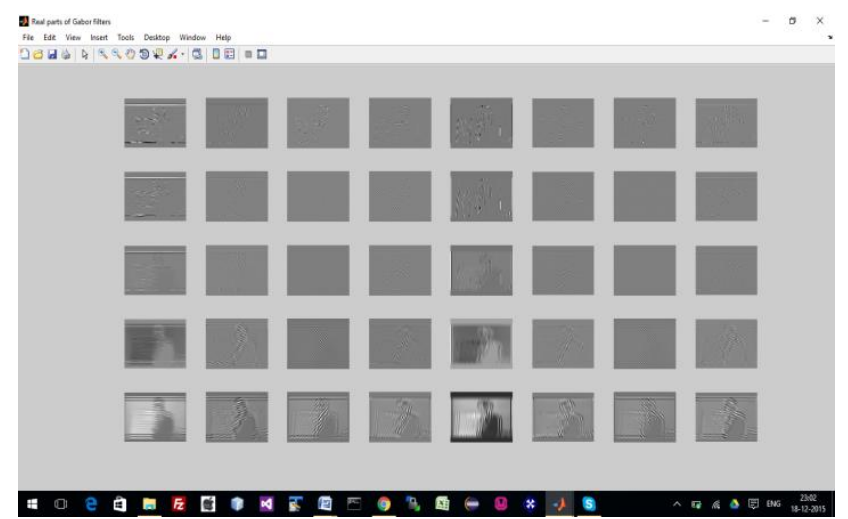

Figure 5.7: GUI Showing the Gabor Featured Images drawn after application of Gabor Filter on the Input Image

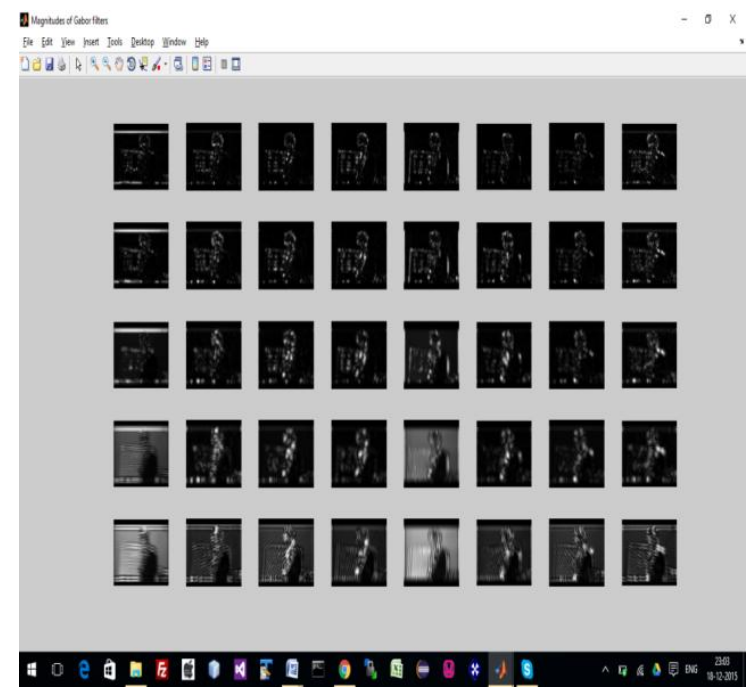

Figure 5.8: GUI Showing the Gabor Featured Images drawn after application of Gabor Filter on the Input Image by edgedetection

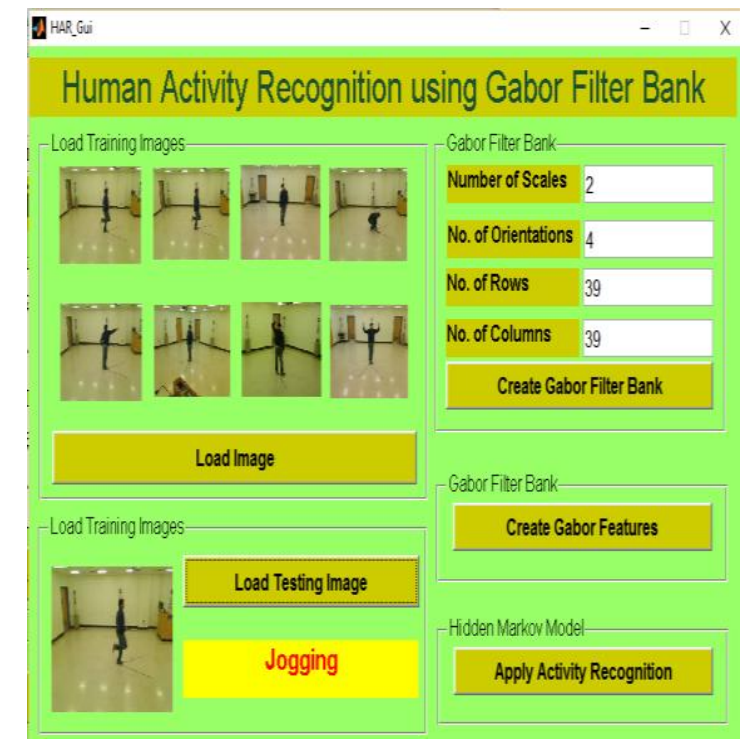

Figure 5.9: GUI Showing the Results obtained on basis of Gabor Feature Extraction, HMM and Viterbi Distance
The proposed device gives immoderate performance with accuracy upto eighty $\%$ i.e for exquisite finding out photos the implementation completed using MATLAB affords accuracy upto eighty two $\%$. The algorithm has been carried out for unique parameter values of filter of Gabor i.e. quantity of scales, extensive form of orientations, variety of rows and extensive form of columns.

\subsection{KTHDataset:}

ג

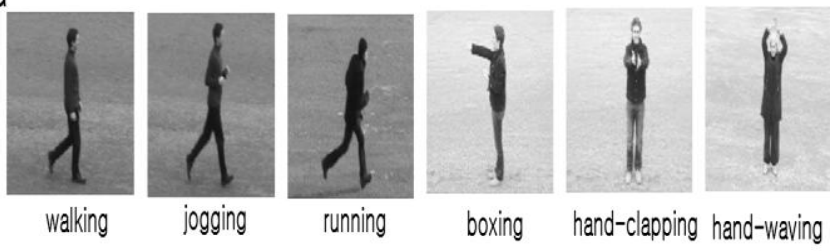

Fig 5.8

Fig 5.eight: within the subsequent, I initially provide the consequences depicting the presentation of the suggested technique and then evaluate our technique to the today's to be had using KTH datasets [7]. The primary experimentation is accomplished on $\mathrm{KTH}$ dataset that is a ordinary fashionable for movement identification with six human motion categories: on foot, walking, jogging, boxing, hand-waving and hand- clapping. every act is presented severa occasions via 25 topics. This dataset holds 599 video collection by means of four numerous scenarios (outside, outdoors with scale variant, exterior by diverse clothes and interior) but with homo- beneficiant and static backgrounds in most collection. In fig 5.eight one character regularly does one motion in all video series.

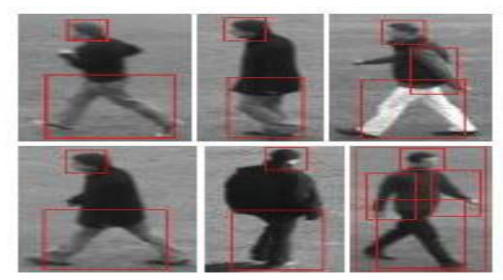

Fig 5.9

we've got used KTH's [7] Database to test the presentation of our machine structure. Fig 5.nine suggests a few detection case of KTH database. inside the KTH's database there are numerous styles of moves: strolling, strolling, boxing, hand waving and so on. the ones moves have been done via using 25 severa humans of similarly sexes in 4 numerous eventualities: outdoors, outdoor with scale variation, outdoor with diverse garments and interior. we've got carried out our technique for all the ones sequences for movement detection.

Desk 1 indicates the assessment of popularity accuracy with distinctive method e.g. nearby function with SVM [7] and factor smart Hidden Markov model of frame factors [12] based totally motion detection. Our method executes high-quality in running, walking; waving and clapping.

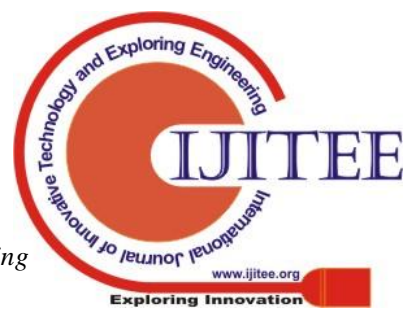


Table 1 represents this graph established in fig five.10. And table 2 gift the common recognition correctness of succession stage over all lessons (taking walks, taking walks, strolling, boxing, waving and clapping of our approach and new modern techniques in KTH data set.

Desk 1: comparison of popularity accuracy with different strategies. Column (a) is neighborhood function and SVM based detection [7], (b) is factor sensible HMM of frame elements [12] primarily based movement detection and(c) is our method

\begin{tabular}{|c|c|c|c|c|}
\hline $\begin{array}{c}\text { No of } \\
\text { classes x }\end{array}$ & Category & A & B & C \\
\hline 1 & Walking & 83.8 & 100 & 100 \\
\hline 2 & Jogging & 60.4 & 60 & 97 \\
\hline 3 & Running & 54.6 & 76.9 & 96.3 \\
\hline 4 & Boxing & 97.9 & 100 & 100 \\
\hline 5 & Clapping & 59.7 & 73.4 & 100 \\
\hline 6 & Waving & 73.6 & 66.7 & 94 \\
\hline
\end{tabular}

Table three indicates the confusion matrix of our technique. on this matrix we will study pass over class with other moves. due to the fact our method don't forget the stochastic vary of body parts which has the main component in movement so if right here is numerous resemblance in motion of these parts in various movements then popularity by way of HMM grow to be complex. we've create numerous of jogging series are misclassified as taking walks and many others.running and running legs are very similar and in some instances there are problems of resolution and comparison Fig 5.10 so it's miles tough to be distinguished. movements concerning hand motion have similar troubles, like some agent acting waving just like clapping, causing ambiguity. The benefit of the our technique is that the detection of body components could be very sturdy and in a check series if detection fails in certain frames then also HMM can detect the movement when you consider that in an extended chain of series average action topology is maintained.

Table 2 Comparison of our method with state-of-the-art methods for KTH dataset

\begin{tabular}{|l|l|}
\hline Method & Accuracy\% \\
\hline Yuan et al[14] & 93.3 \\
\hline Sunil et al [13] & 94 \\
\hline Kovashka et al. [16] & 94.54 \\
\hline Atomosukartor et al. [18] & 95.37 \\
\hline Wu etal. [17] & 95.7 \\
\hline Jeongmin Yu etal. [19] & 96.3 \\
\hline Our approach(Gabor filter with HMM) & 97.8 \\
\hline
\end{tabular}

Fig 5.10 direction garage bins same to 8.

\begin{tabular}{|c|l|l|l|l|l|l|}
\multicolumn{7}{|c}{ Gabor Filter with HMM } \\
\hline Category & Walking & Jogging & Running & Boxing & Clapping & Waving \\
\hline Walking & 100 & 0 & 0 & 0 & 0 & 0 \\
\hline Jogging & 3 & 97 & 0 & 0 & 0 & 0 \\
\hline Running & 0 & 3.7 & 96.3 & 0 & 0 & 0 \\
\hline Boxing & 0 & 0 & 0 & 100 & 0 & 0 \\
\hline Clapping & 0 & 0 & 0 & 100 & 0 & 0 \\
\hline Waving & 0 & 0 & 0 & 0 & 06 & 94 \\
\hline
\end{tabular}
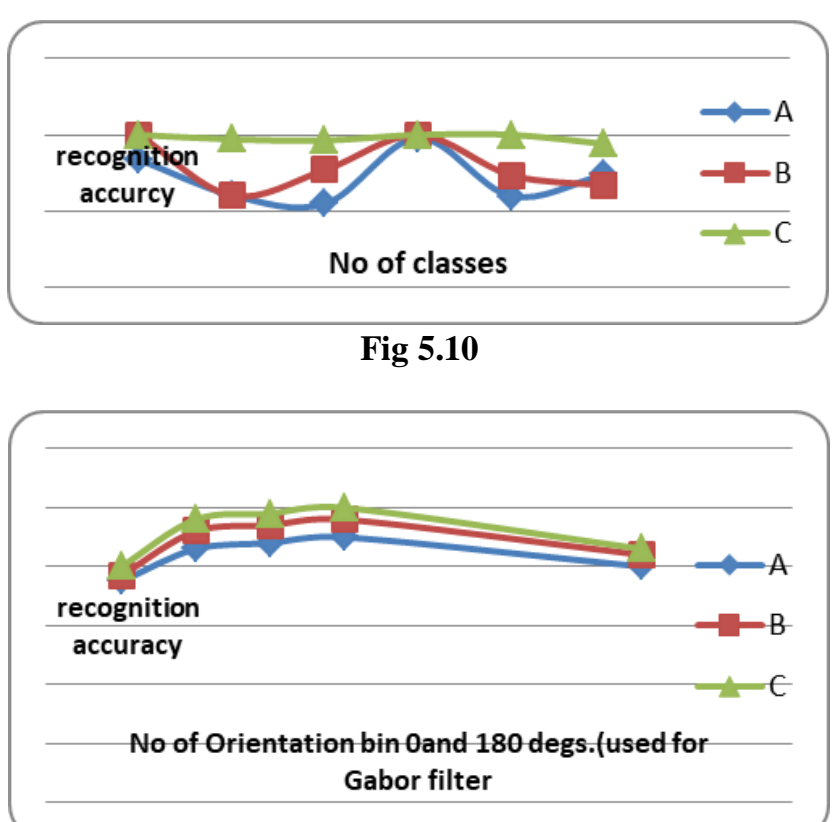

Fig.5.11 Test Accuracy obtained with changing quantity of direction bins in Gabor filter banks for different grid sizes

Determine 5.11 depicts the distinction of the recognition price due to the truth the huge sort of orientation-boxes (amongst 0 and a hundred and 80 degrees) in the clear out economic group of Gabor is progressed. whilst the form of orientation-boxes are very small (collectively with 2), the Gabor skills are not able to seize the actual orientation well that is pretty inexpensive because the underlying actual orientations of body-additives may be some distance from those boxes. as soon as it will increase to a higher price (which include 6 or eight), it captures the ideal orientations of frame-elements. The abilities pass over proper orientations once more whilst too many containers are used (which include 16) as there aren't enough pixels vote casting for a specific orientation. discern 3 moreover indicates the equal graphs for severa grid sizes. real grid length is predicated upon at the relative characteristic of the digital virtual camera and consequently the intensity of the devices. therefore, in a everyday surveillance software softwaresoftware program, you can determine it a priori for traditional situations. We got the nice outcomes with the useful resource of selecting $4 \times 4$ or $8 \times 8$ grids and amount of 


\section{CONCLUSIONS AND FUTURE DIRECTION}

In this article we superior human interest verification tool: a HMM - based totally absolutely tool. The characteristic extraction technique is based on the functions doing away with of GABOR and the computation of the DRT. For this scheme Gabor clear out is used for deciding on Gabor characteristic for the face recognition. A small subnet of abilities of Gabor in a position of selective workplace work particular human hobby pix which may be saved in the database. on this paper the approach superior rent the hidden Markov model to in form a check human interest picture with a suitable reference photo. This device use clean out of Gabor to dispose of the gathering of informative Gabor characteristic from the given human hobby image to extracted characteristic are all once more subjected to DRT to extracted functions vectors from a photo the HMM-based totally totally scheme superior in this newsletter identical the set of characteristic (assertion collection) for the take a look at photo with the HMM of claimed photograph, in the end of vitrerbi affiliation. the same operation is capable of be completed through way of using log Gabor easy out with HMM and development of diploma detection method can be superior

\section{REFERENCES}

1. Barua, S. (1992). Neural Networks and their Applications to Computer Security. Proceedings of the SPIE - International Society for Optical Engineering, pp. 735-742

2. Bracewell, R.N. (1995). Two-Dimensional Imaging. Prentice Hall

3. Bastos, L.C., Bortolozzi, F., Sabourin, R., and Kaestner, C. (1997). Mathematical Modelation of Handwritten Signatures by Conics. Revista da SociedadeParanaese de Matem'atica, vol. 18 , pp. 135-146

4. J. Davis and S. Taylor. Analysis and recognition of walking movements. In Analysis and recognition of walking movements, Quebec, Canada, pages 11-15, 2002

5. Coetzer, J., Herbst, B.M., and Du Preez, J.A. (2004). Offline Signature Verification Using the Discrete Radon Transform and a Hidden Markov Model. Eurasip Journal on Applied Signal Processing - Special Issue on Biometric Signal Processing, Bourland,

6. H., Pitas, I., Lam, K. K., and Wang, Y., editors, vol. 2004, no. 4, pp. 559-571.

7. C. Schuldt, I. Laptev, and B. Caputo. Recognizing human actions: a local svm approach. In ICPR III, pages 32-36, 2004

8. A. Micilotta, E. Ong, and R. Bowden. Detection and tracking of humans by probabilistic body part assembly. In British Machine Vision Conference, pages 429-438, 2005

9. T. Moeslund, A. Hilton, and V. Kruger". A survey of advances in vision-based human motion capture and analysis. In CVIU 104, pages 90-126, 2006

10. L. Fengjun and N. Ramkant. Recognition and segmentation of 3-d human action using $\mathrm{hmm}$ and multi-class adaboost. In Computer Vision-ECCV, pages 359-372, 2006

11. D. Ramanan, D. Forsyth, and A. Zisserman. Tracking people by learning their appearance. IEEE Transaction on PAMI, 29(1):65-81, 2007

12. BhaskarChakraborty, OgnjenRudovic Gonzalez. ViewInvariant Human-Body Detection with Extension to Human
Action Recognition using Component-Wise HMM of Body Parts 2008 IE

13. Sunil Kumar Gupta, Y Senthil Kumar and K R Ramakrishnan. Learning Feature Trajectories Using Gabor Filter Bank for Human Activity Segmentation and Recognition ICVGIP, pages 111-118 2008

14. J.Yuan, Z.Liu, Y.Wu, Discriminative subvolume search for efficient action detection, in: Proceedings of the Conference on Computer Vision and Pattern Recognition, 2009, pp.24422449

15. Rajeev Shrivastava\&Ankitanigam "Analysis and performance of face recognition system using Gabor filter bank with HMM model "Proceedings of the InternationalConference on TRENDZ IN INFORMATION SCIENCES \& COMPUTING (TISC 2010 )IEEE 2010

16. A.Kovashk andK.Grauman, Learning a hierarchy of discriminative space-time neighborhood features for human action recognition ,in: Proceedings of the Conference onComputer Vision and Pattern Recognition, 2010,pp.20462053.

17. S.Wu, O.Oreifej, M.Shahet.all Action recognition in videos acquired by a moving camera using motion decomposition of Lagrangian particle trajectories, in: Proceedings of the International Conference on Computer Vision, 2011, pp.1419-1426

18. Atomosukartor, B.Ghanem, N. Ahuja, Trajectory-based fisher kernel representation for action recognition in videos, in: IEEE International Conference on Pattern Recognition,2012,pp.3333-3336.

19. Jeongmin $\mathrm{Yu}$ a, MoonguJeona,n, WitoldPedrycz Weighted feature trajectories and concatenated bag-of-features for action recognition Neurocomputing (2013), 10.1016/j.neucom.2013.10.024

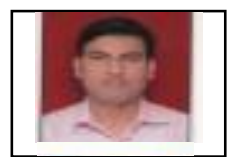

BIBLIOGRAPHY

DrRajeev Shrivastava is working as a HOD in St. Martin's Engg. College He has done $\mathrm{PhD}$ in Digital Image Processing from JVWU Jaipur in 2016.He is presently doing PhD in Digital Image Processing from JVWU Jaipur. He has published 36 international paper and 3 books. He also got young scientist award in 2017. 